\title{
Requisitos globales mínimos esenciales en educación médica*
}

Comité Central, Instituto para la Educación Médica Internacional (IIME), New York, USA.**

\section{Resumen}

El proceso de la globalización, cada día más evidente en la educación médica, ha convertido en asunto urgente la tarea de definir las capacidades esenciales que requieren los "médicos globalizados". De este asunto se ha ocupado el recientemente creado Instituto para la Educación Médica Internacional, IEMI (Institute for International Medical Education IIME). El Comité Central del IEMI desarrolló el concepto de "requisitos globales mínimos esenciales" (RGME) y definió un conjunto de resultados mínimos del aprendizaje, aplicables en todo el mundo, que los estudiantes de las escuelas (facultades) de medicina deben demostrar para poder obtener su grado. Estos "requisitos esenciales" se agrupan en siete grandes categorías educacionales y comprenden $\mathbf{6 0}$ objetivos de aprendizaje. Además de estas "capacidades globales", las escuelas de medicina deben agregar requisitos nacionales y locales. El enfocar las capacidades de los estudiantes como resultados de la educación médica debe tener implicaciones profundas en el contenido curricular y en los procesos educacionales de las escuelas (facultades) de medicina. \footnotetext{
del IIME.

** Correspondencia:

Dr. Andrzej Wojtczak,

Institute for International Medical Education,

106 Corporate Park Drive, Suite 100,

White Plains, NY 10604-3817, USA.

Tel: (1)914-253-6633;

Fax: (1)914-253-6644;

E-mail: wojtczak@iime.org
}

* Traducción del Dr. Juan Mendoza Vega. Presidente Academia Nacional de Medicina de Colombia. "Medical Teacher, 2002, vol.24(2):130-155 (www.tandf.co.uk/journals)", con autorización

\section{INTRODUCCIÓN}

La Junta Directiva del China Medical Board of New York, Inc., aprobó el 9 de junio de 1999 una donación para fundar el Instituto para la Educación Médica Internacional IEMI. Es tarea del Instituto liderar la definición de "requisitos globales mínimos esenciales" para los programas de pregrado en educación médica. Estos "requisitos esenciales" deben consistir en conocimientos médicos, habilidades clínicas, actitudes profesionales, características de comportamiento y de ética que todos los médicos deben tener, sea cual fuere el lugar donde recibieron su formación.

La tarea de definir esos "requisitos globales mínimos esenciales" se entregó al Comité Central, conformado por expertos de talla internacional en educación médica, de todas las partes del mundo. La Junta Directiva del IEMI, en el que tienen asiento ocho expertos de larga trayectoria en educación médica y políticas de salud, supervisa las labores del Instituto y ayuda a guiar las labores del Comité Central, al cual llegan también sugerencias del Comité Consultor del IEMI que está formado por los Presidentes o representantes especiales de 14 grandes organizaciones internacionales involucradas en la educación médica. Se tiene así un foro en el cual se intercambia información, se escuchan sugerencias y se colabora para que los esfuerzos en el seno del IEMI sean complementarios y no contradictorios.

Desde el principio se comprendió con claridad que la definición de tales capacidades o resultados del proceso de educación médica tendría implicaciones significativas para el currículo en las escuelas (facultades) de medicina; quienes quisieran obtener en ellas su grado, deberían demostrar capacidades 
profesionales que a su vez aseguraran la excelente calidad de sus servicios, unida a la necesaria empatía y el respeto por el bienestar de los pacientes.

Dentro de este marco, los graduandos deben ser capaces de integrar el tratamiento de las enfermedades y de las lesiones, con la promoción de la salud y la prevención, además de poder trabajar en equipos multi-profesionales. También deben saber enseñar, aconsejar y guiar a los pacientes, a las familias y a la comunidad en las cuestiones relacionadas con la salud, las enfermedades, los factores de riesgo y los "estilos" saludables de vida. Deben tener la capacidad para adaptarse a los cambios en las características de las enfemedades, a los nuevos requisitos y condiciones de la práctica médica, a la tecnología de la información médica, a los avances de la ciencia y a los cambios en los sistemas de cuidados para la salud, pero siempre manteniendo los más elevados estándares en la ética y los valores de la profesión.

El proyecto del IEMI tiene tres fases:

La primera (Fase I), "Definición de los requisitos esenciales", comenzó con la creación del Instituto para la Educación Médica Internacional. Su tarea era desarrollar un conjunto de "requisitos globales mínimos esenciales" (RGME) en los que se aprovecharían parcialmente algunos estándares ya existentes. Tales estándares incluían las ciencias básicas en medicina, experiencias clínicas, conocimientos, habilidades, valores profesionales, modelos de conducta y valores éticos. Los "requerimientos esenciales" debían entenderse sólo como el núcleo de un currículo médico, por cuanto cada país, región y escuela (facultad) de medicina tienen sus propios requerimientos que deben incorporarse al currículo particular; esto significa que el programa educacional de cada escuela debe ser diferente de los otros pero teniendo siempre un núcleo común e igual para todos.

En la segunda fase (Fase II), la "Implementación Experimental" de los RGME se utilizará para evaluar a los graduados de las principales escuelas (facultades) de medicina en China. Dichas escuelas utilizarán los métodos de evaluación que son adecuados para su experiencia, cubrirán todas las siete grandes categorías y los 60 objetivos de aprendizaje, y al participar en el experimento buscarán identificar las fortalezas y las fallas que puedan existir en ellas. Luego, se realizarán los esfuerzos conducentes a mejorar las áreas que se encuentren débiles, antes de realizar una segunda evaluación. Si una escuela (facultad) cumple con todos los "requisitos esenciales", se le otorgará la certificación correspondiente.
Durante la tercera fase (Fase III) o "Fase de diseminación", tanto el proceso seguido como las enseñanzas que éste haya dejado se modificarán como sea necesario y se pondrán a disposición de cuantos realizan educación médica en todo el mundo, para su utilización. Se tiene la esperanza de que los "requisitos esenciales" sirvan como herramienta para mejorar la calidad de la educación médica y den las bases para el establecimiento de un sistema internacional de valoración de los programas de educación médica.

\section{ANTECEDENTES}

Los impulsos de globalización se hacen cada vez más evidentes en la educación médica. Esto parece muy lógico si se tiene en cuenta que la medicina es una profesión en todo el mundo y que por tradición, la investigación y los conocimientos en el terreno médico han traspasado las fronteras entre naciones. Ha sido costumbre que los médicos puedan estudiar su profesión y prestar sus servicios en países diversos; además, la creatividad humana exige que la globalización incluya actividades intelectuales y culturales. Diversos convenios y tratados multilaterales están abriendo las puertas a la posibilidad de que las personas se movilicen por todo el mundo, así como estimulan el establecimiento de estándares educacionales comunes, el reconocimiento mutuo de calificaciones y la adopción de procesos de certificación mediante los cuales se permita a los profesionales practicar su vocación en sitio distinto de aquel en que se formaron.

En este momento hay cerca de seis millones de médicos en todo el mundo, que prestan sus servicios a más de seis mil millones de habitantes. Esos médicos reciben su educación y entrenamiento en unas 1800 escuelas (facultades) de medicina en todo el planeta; y aunque a primera vista todos los currícula parecen similares, en realidad hay gran variación entre sus contenidos. Se han realizado, con éxito parcial, algunos intentos por evaluar los procesos que llevan a un grado de doctor en medicina (MD) o su equivalente, pero pocos de esos intentos se han enfocado en los resultados de los esfuerzos educacionales. En realidad, nunca se ha tratado de definir las capacidades fundamentales o mínimas que un médico debe tener cuando termina sus estudios de pregrado, antes de entrar a la etapa de estudios de post-grado o especialización; además, en algunos países se ha presentado proliferación de nuevas escuelas de medicina sin que al 
mismo tiempo se hayan puesto en marcha los mecanismos adecuados para asegurar la calidad de la educación médica.

De manera simultánea, tanto los servicios de salud como la práctica de la medicina están sufriendo cambios profundos, forzados por las dificultades económicas para la financiación de los sistemas de cuidado para la salud. El costo creciente de las intervenciones en salud y las correspondientes políticas de contención de costos, pueden poner en peligro el humanismo y los valores de la Medicina. En consecuencia, es necesario preservar las metas de equidad y de beneficio social frente a las crecientes presiones y barreras de tipo económico.

En las ciencias biomédicas, la tecnología de la información y la biotecnología se están presentando rápidos progresos; ellos plantean nuevos problemas éticos, sociales y legales para la profesión médica y exigen que se conserve el equilibrio entre Ciencia y Arte en la Medicina. Una tarea importante para la educación médica es preparar a los futuros doctores de modo que sean capaces de adaptarse a las condiciones de esa práctica médica en un ambiente de cuidados a la salud tan rápidamente cambiante. El reto que tiene ante sí la comunidad educacional en medicina, es el de utilizar la globalización como instrumento oportuno para mejorar la calidad de la educación y de la práctica médicas.

Al definir las capacidades esenciales que todo médico debe tener, se necesita poner énfasis especial en el profesionalismo, las ciencias sociales, la economía de la salud, el manejo de la información y los sistemas de cuidados a la salud. Esto debe hacerse en el contexto de las características sociales y culturales de las diversas regiones del planeta. Los métodos precisos y la forma de enseñar pueden variar de una escuela a otra, pero las capacidades exigidas deben ser las mismas en todas. Es claro que el concepto de "requisitos esenciales" no implica la uniformidad global de los currícula ni de los procesos educacionales en medicina; tampoco son estos requisitos globales esenciales una amenaza contra el principio fundamental de que la educación médica debe identificar necesidades específicas sociales y culturales, en el contexto del lugar donde el médico se forma y donde va a ejercer, y responder a esas necesidades. Al tratar de cumplir con los "requisitos globales mínimos esenciales" las escuelas (facultades) de medicina adoptarán cada una su propio diseño curricular, pero al hacerlo, deberán asegurarse de que sus graduados hayan adquirido las capacidades básicas que se señalan en los "requerimientos

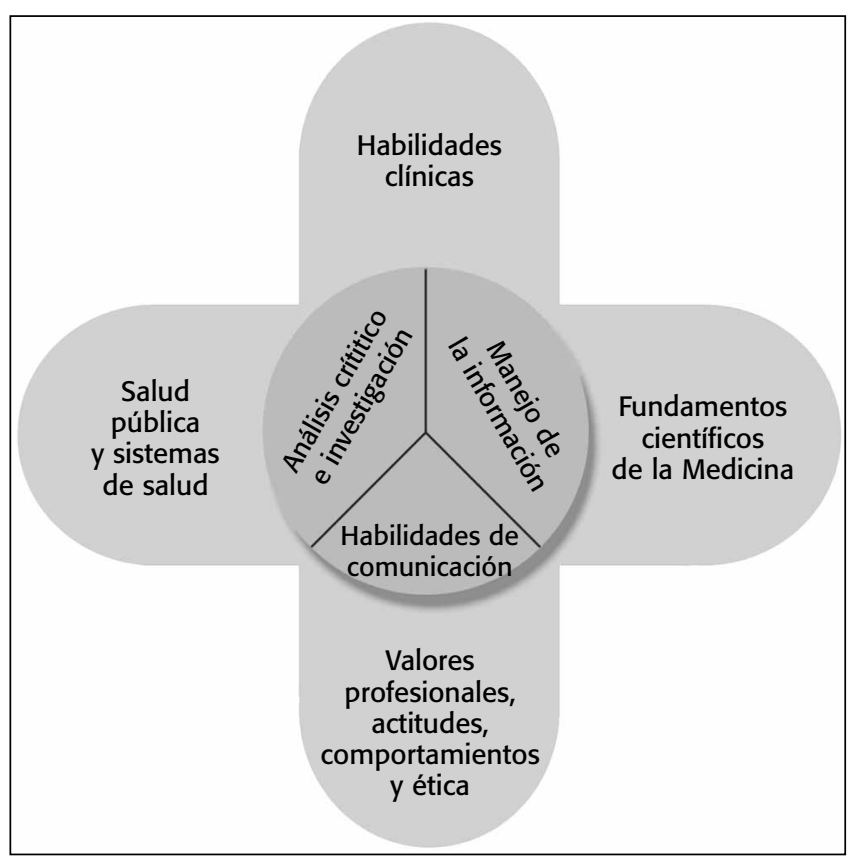

Fig. 1. Categorias de los requisitos esenciales

mínimos esenciales". En pocas palabras, deben "pensar globalmente y actuar localmente".

El Comité Central agrupó los "requisitos esenciales" en las siguientes siete grandes categorías de resultados o capacidades educacionales (Figura 1):

\section{VALORES PROFESIONALES, ACTITUDES, COMPORTAMIENTOS Y ÉTICA}

El profesionalismo y la conducta ética son indispensables para la práctica de la Medicina. El profesionalismo incluye no sólo conocimientos y habilidades médicas, sino el compromiso de respetar un conjunto de valores compartidos con los colegas, la autonomía para señalar y hacer respetar esos valores, y las responsabilidades inherentes a ellos. El graduado en medicina debe demostrar:

- Reconocimiento y comprensión de los elementos esenciales de la profesión médica, incluyendo los principios éticos y morales y las responsabilidades legales que implica la profesión.

- Valores profesionales, que incluyen la excelencia, el altruismo, el sentido del deber, la compasión, la empatía, la responsabilidad, la honestidad y la integridad, así como el sometimiento al método científico.

- Comprensión de la obligación que tiene cada médico, en cuanto a la promoción, protección e incremento de estos elementos esenciales, para beneficio de los pacientes, de la profesión y de la Sociedad en general. 
- Comprensión de que la buena práctica médica depende del mutuo entendimiento y relación entre el médico, el paciente y la familia, en cuanto al respeto por el bienestar del enfermo, la diversidad cultural las creencias y la autonomía de las personas.

- Habilidad para aplicar los principios del razonamiento moral y de la toma de decisiones a los conflictos que puedan surgir entre lo ético, lo legal y los intereses profesionales en las diversas situaciones, incluyendo los aspectos planteados por las restricciones económicas, la comercialización del cuidado a la salud y los avances científicos.

- Autoregulación y la conciencia de que es necesario su mejoramiento personal, así como reconocimiento de sus propias limitaciones y las limitaciones en sus conocimientos médicos.

- Respeto por sus colegas y por los demás profesionales del área de la salud, y habilidad para estimular relaciones positivas de colaboración con ellos;

- Comprensión de la obligación moral de dar cuidados adecuados en los momentos finales de la vida, incluyendo la suficiente paliación de los síntomas que puedan causar sufrimiento al enfermo llegado a tal extremo.

- Reconocimiento y comprensión de los aspectos éticos y médicos que existen en el secreto profesional, el manejo de la documentación de los pacientes, el plagio y la propiedad intelectual.

- Habilidad para planear de manera efectiva y manejar con eficiencia su propio tiempo y actividades para enfrentar lo aleatorio, y habilidad para adaptarse a los cambios.

- Admisión de responsabilidad personal en el cuidado de las personas enfermas.

\section{FUNDAMENTOS CIENTÍFICOS DE LA MEDICINA}

El graduado debe poseer los conocimientos requeridos para contar con sólidos fundamentos científicos médicos, así como ser capaz de aplicar esos conocimientos a la solución de problemas médicos concretos; debe entender los principios en que se basan las decisiones y actos médicos, y ser capaz de adaptarse al cambio según lo vayan requiriendo el paso del tiempo y el contexto de su propia práctica. Para lograr tales resultados, el graduando debe demostrar conocimiento y comprensión de:

- La estructura y función normal del cuerpo humano como sistema biológico complejo y adaptativo.

- Las anormalidades que la enfermedad produce en la estructura y las funciones del cuerpo.

- La conducta humana, normal y anormal.
- Los determinantes y los factores de riesgo importantes para la salud y la enfermedad, la interacción entre el ser humano y el ambiente físico y social que lo rodea.

- Los mecanismos moleculares, celulares, bioquímicos y fisiológicos que mantienen la homeostasis corporal.

- El ciclo de la vida humana y los efectos del crecimiento, el desarrollo y el envejecimiento tanto sobre el individuo como sobre la familia y la comunidad.

- La etiología y la historia natural de las enfermedades agudas y crónicas.

- La epidemiología, la economía de la salud y la gestión en salud.

- Los principios de acción de los medicamentos, sus usos y eficacia en los diversos tratamientos.

- Las intervenciones pertinentes de tipo bioquímico, farmacológico, quirúrgico, psicológico, social o de otras clases, tanto para las enfermedades agudas o crónicas como para la rehabilitación y para los cuidados al final de la vida.

\section{HABILIDADES DE COMUNICACIÓN}

El médico debe crear un ambiente en el cual esté presente el aprendizaje mutuo entre los pacientes, sus parientes, los miembros del equipo profesional de salud y los colegas médicos, así como el público en general, mediante una comunicación eficiente. Para incrementar las posibilidades de apropiadas decisiones por parte de los médicos y de satisfacción de los pacientes, los graduandos deben ser capaces de:

- Escuchar con atención, obtener y sintetizar información pertinente acerca de los problemas que aquejan al enfermo, y comprender el contenido de esa información.

- Aplicar habilidades de comunicación para facilitar la comprensión con los enfermos y sus familias, $\mathrm{y}$ ayudarlos a tomar decisiones en condiciones de igualdad humanitaria.

- Comunicarse adecuadamente con los colegas, los profesores, la comunidad, otros grupos y los medios de comunicación social.

- Interactuar con otros profesionales involucrados en la atención a los enfermos, mediante eficiente trabajo en equipo.

- Demostrar capacidades básicas y actitud positiva en cuanto se refiere a educar y enseñar a otras personas.

- Demostrar sensibilidad hacia los factores culturales y personales que pueden mejorar las interacciones con los pacientes y la comunidad. 
- Comunicarse de modo efectivo, tanto oralmente como por escrito.

- Elaborar y mantener buenas historias clínicas y otros registros médicos.

- Sintetizar y presentar información apropiada a las necesidades de una audiencia determinada, discutir planes realizables y aceptables de acción para enfrentar los asuntos que sean prioritarios para un individuo o una comunidad.

\section{HABILIDADES CLÍNICAS}

El graduado debe poder diagnosticar y disponer el tratamiento de pacientes en forma eficiente y efectiva. Para ello, debe ser capaz de:

- Elaborar una historia clínica apropiada, que incluya los aspectos sociales y los de salud ocupacional;

- Realizar un examen físico y mental.

- Aplicar técnicas y procedimientos básicos de diagnóstico, analizar e interpretar los resultados que obtenga y definir la naturaleza del problema al cual se enfrenta.

- Llevar a cabo estrategias adecuadas de diagnóstico y tratamiento, especialmente los procedimientos urgentes para salvar la vida, y aplicar los principios de la medicina basada en la mejor información disponible.

- Ejercer buen juicio clínico para establecer diagnósticos y ordenar tratamientos.

- Reconocer sin demora las situaciones que ponen la vida en peligro inmediato.

- Tratar las emergencias médicas más frecuentes;

- Tratar los pacientes de manera ética, efectiva y eficiente, sin dejar de lado la promoción de la salud y la prevención de la enfermedad.

- Valorar los problemas de salud y aconsejar lo que convenga a los pacientes, teniendo en cuenta los factores orgánicos, psicológicos, sociales y culturales.

- Comprender el uso apropiado de los recursos humanos, las intervenciones para diagnóstico, las modalidades terapéuticas y los medios materiales para el cuidado de la salud.

\section{SALUD PÚBLICA Y SISTEMAS DE SALUD}

Los graduados de Medicina deben entender el papel que les corresponde en la protección y promoción de la salud en las comunidades humanas, y ser capaces de realizar las acciones adecuadas. Deben comprender los principios de organización de los servicios de salud, sus fundamentos económicos y legales. También deben tener conocimientos básicos sobre la gestión eficiente y efectiva de sistemas de salud. Los graduandos deben demostrar.

- Conocimiento de los determinantes de salud más importantes en una comunidad, tanto los dependientes del estilo de vida como los genéticos, demográficos, ambientales, sociales, económicos, psicológicos y culturales.

- Conocimiento de su papel y de sus habilidades para llevar a cabo las acciones apropiadas para la prevención y protección ante enfermedades, lesiones o accidentes, mantenimiento y promoción de la salud, tanto de los individuos como de las familias y de la comunidad.

- Conocimiento de la situación internacional en el campo de la salud, de las tendencias globales de morbilidad y mortalidad en enfermedades crónicas de impacto social, de la influencia de las migraciones, los intercambios comerciales y los factores ambientales sobre la salud, y el papel de las organizaciones internacionales de salud.

- Aceptación de los papeles y responsabilidades de otros profesionales de la salud o relacionados con asuntos de salud, en cuanto al suministro de cuidados a la salud para personas, grupos de población y comunidades.

- Comprensión de la necesidad de compromiso colectivo en las intervenciones para promoción de la salud, cuando éstas requieren participación de la población a la cual se dirigen, y de la necesidad de enfoques multidisciplinarios que incluyan a los diversos profesionales de la salud y también a colaboradores de otros sectores.

- Comprensión de las cuestiones básicas en los sistemas de salud, entre ellas sus políticas, organización, financiación, medidas de contención de costos y principios de gerencia efectiva en el suministro de atención a la salud.

- Comprensión de los mecanismos que determinan la equidad en el acceso a los cuidados de salud, la efectividad y calidad de esos cuidados.

- Capacidad para utilizar los datos nacionales, regionales y locales de vigilancia demográfica y epidemiológica, al tomar decisiones sobre salud.

- Voluntad para aceptar el liderazgo y la dirección de otras personas, cuando sea necesario y apropiado, en cuestiones de salud.

\section{MANEJO DE LA INFORMACIÓN}

La práctica de la medicina y la gestión de sistemas de salud requiere el flujo efectivo de información y de conocimientos. Los progresos en la tecno- 
logía de la computación y la comunicación se han concretado en poderosos instrumentos para la educación y para el análisis y manejo de la información. Por ello, los graduandos deben comprender las posibilidades y limitaciones que tienen la tecnología de la información y el manejo del conocimiento, y ser capaces de utilizar todo ello para resolver problemas y tomar decisiones en el campo médico. El graduado debe ser capaz de:

- Buscar, recolectar, organizar e interpretar infor-mación biomédica y de salud, en diversas bases de datos y otras fuentes de información.

- Recuperar información específicamente relacionada con pacientes individuales, en un sistema de datos clínicos.

- Utilizar la tecnología de la información y la comunicación para ayudar al diagnóstico, la terapéutica y las medidas de prevención, así como para supervisar y controlar situaciones de salud.

- Comprender la aplicación y las limitaciones de la tecnología de la información.

- Mantener registros adecuados de su práctica, para efectos de análisis y mejoramiento de ella.

\section{ANÁLISIS CRÍTICO E INVESTIGACIÓN}

La habilidad para valorar en forma crítica los conocimientos existentes, la tecnología y la información, es indispensable para la resolución de problemas, porque los médicos deben adquirir continuamente nueva información científica y nuevas habilidades, para poder mantener su capacidad profesional. La buena práctica de la Medicina requiere la habilidad para pensar científicamente y usar el método científico. En consecuencia, el graduando en medicina debe ser capaz de:

- Demostrar en sus actividades profesionales un punto de vista crítico, creatividad, escepticismo constructivo y actitud orientada hacia la investigación;

- Comprender el poder y las limitaciones del pensamiento científico basado en la información obtenida de diversas fuentes, cuando se trata de establecer la causa y disponer el tratamiento y la prevención de las enfermedades.

- Utilizar su criterio personal para la resolución crítica y analítica de problemas, así como buscar la información en cambio de limitarse solo a esperar que se la suministren otros.

- Identificar, formular y resolver problemas de los pacientes, utilizando el pensamiento científico y tomando como base la información obtenida de diversas fuentes y adecuadamente correlacionada.
- Comprender el sitio que tienen la complejidad, la incertidumbre y las probabilidades, en las decisiones de la práctica médica.

- Formular hipótesis, recolectar y valorar críticamente los datos para la solución de problemas.

Para mantener y mejorar las capacidades adquiridas en la escuela (facultad) de medicina, los graduandos deben estar conscientes de sus propias limitaciones, de la necesidad de autoevaluación repetida a intervalos regulares, de la aceptación de valoración por parte de sus pares, así como de la costumbre de estudiar continuamente por su propia voluntad. Tales actividades de desarrollo personal permiten la adquisición continua de nuevos conocimientos y habilidades, para uso en su carrera profesional.

Los "requisitos esenciales" por sí solos, probablemente no cambiarán las capacidades de los graduados a menos que junto con ellos se realice evaluación de las capacidades de los estudiantes. Por ello, para poner en acción este documento es indispensable tener herramientas de evaluación de los resultados educacionales. Así se tendrá la certeza de que los graduados en Medicina, cualquiera que sea el lugar del mundo donde hayan sido formados, tendrán capacidades básicas similares en el momento de iniciar estudios más avanzados, de postgrado (formación como especialistas) o al iniciar su práctica (como médicos generales) bajo la supervisión que en cada país determinen las autoridades nacionales. Un equipo específico del IEMI, “Task Force”, está trabajando en el desarrollo de las mencionadas herramientas.

Los "Requisitos Globales Mínimos Esenciales" que se acaban de presentar en este documento, se consideran instrumento para mejorar la calidad de la educación médica e, indirectamente, de la práctica médica. Se espera que este proyecto del IEMI tenga influencia significativa sobre los currícula de las escuelas (facultades) de medicina y sobre los procesos educacionales, abriendo así el camino para una educación médica orientada hacia la obtención demostrable de capacidades.

\section{NOTAS SOBRE LOS COLABORADORES}

ELIZABETH G. ARMSTRONG, Directora de Educación Médica, Harvard Medical School, Boston, MA., EUA.

RAJA C. BANDARANAYAKE, profesor y jefe del Departamento de Anatomía, Universidad del Golfo Árabe, Colegio de Medicina y Ciencias Médicas, Manama, Bahrain.

ALBERTO ORIOL I BOSCH, Director del Instituto de 
Estudios de Salud, Departamento de Salud y Seguridad Social del Gobierno Catalán, Barcelona, España.

ALEJANDRO CRAVIOTO, Decano de la Facultad de Medicina, Universidad Nacional Autónoma de México, Ciudad de México.

CHARLES DOHNER, Profesor Emérito de Educación Médica, Universidad de Washington, Seattle, EUA.

MARVIN R. DUNN, Presidente del Comité Central del Instituto para la Educación Médica Internacional y Director del Comité de Actividades de Control sobre las Residencias, Consejo de Acreditación para la Educación Médica de Post-grado, Chicago, EUA.

JOSEPH S. GONNELLA, Director del Centro para la Investigación sobre Educación Médica y Cuidado de la Salud, y Decano emérito del Jefferson Medical College, Philadelphia, EUA.

JOHN D. HAMILTON, Director académico del Currículo de Pre-grado, Universidad de Durham, Campus de Stockton, Stockton-on-Tees, UK y antes Profesor de Medicina en la Universidad de Newcastle, Australia.

RONALD M. HARDEN, Vicedecano de Educación Médica y director del Centro de Educación Médica, Universidad de Dundee. En la actualidad es también Secretario General de la Asociación para la Educación Médica en Europa, con sede en Dundee, Escocia, UK.

DAVID HAWKINS, Director ejecutivo de la Asociación Canadiense de Colegios Médicos, Ottawa, Canadá.

JOSE FELIX PATIÑO, Presidente de la Academia Nacional de Medicina de Colombia, Profesor Visitante, Yale University, EUA.

M. ROY SCHWARZ, Presidente del China Medical Board of New York, Inc. y profesor en la Universidad de Washington y la Universidad de California en San Diego. Es también Presidente de los Comités de Dirección y Consultivo del IEMI, New York, EUA.

DAVID T. STERN, Presidente de la Fuerza de Tarea para la Evaluación, Instituto para la Educación Médica Internacional. Es también profesor asistente de Medicina y director de Programas Estandarizados para Pacientes, University of Michigan Medical Center, Ann Arbor, MI, EUA.

PRASONG TUCHINDA, Decano, Facultad de Medicina de Rangsit University, y presidente de las Asociaciones de Médicos Generales y de Familia, Bangkok, Tailandia.

J.P. DE V. VAN NIEKERK, Decano emérito, Universidad de Ciudad del Cabo, Cape Town, Suráfrica.

ANDRZEJ WOJTCZAK, Director del Instituto para la Educación Médica Internacional, Nueva York, profesor en la Escuela de Salud Pública y Medicina Social en Varsovia, Polonia. Antes fue director del Centro de Investigación en Salud de la OMS en Kobe, Japón, y presidente de la AMEE.
ZHOU TONGFU, Subdirector del Comité de Educación de la Provincia de Sichuan y profesor en el Centro Médico de la Universidad de Sichuan, Chengdu, República Popular China.

\section{BIBLIOGRAFÍA}

Accreditation Council for Graduate Medical Education (ACGME) (1999) Outcome Project \& General Competencies.

Accreditation and the Liaison Committee on Medical Education (1998) Functions and Structure of a Medical School, Standards for Accreditation of Medical Education Programs Leading to the M.D. Degree (Washington, D.C., Association of Medical Colleges and the American Medical Association).

American Medical Association (1993) The Potential Impact of Health System Reform on Medical Education (Working Group on Medical Education and Health System Reform, Office of Medical Education).

Association of American Medical Colleges (1984) Physicians for the Twenty-First Century, The GPEP Report, Report of The Panel on the General Professional Education of the Physician and College Preparation for Medicine (Washington, D.C., AAMC).

Association of American Medical Colleges and American Medical Association (1997) Guide to the Institutional Self-Study - Program of Medical Education Leading to the MD Degree (Chicago, IL and Washington, D.C., Liaison Committee on Medical Education).

Association of American Medical Colleges and American Medical Association (1998) The Role of Students in the Accreditation of U.S. Medical Education Programs (Chicago, IL and Washington, D.C., Liaison Committee on Medical Education).

Association of American Medical Colleges and American Medical Association (1998) Rules of Procedure (Chicago, IL and Washington, D.C., Liaison Committee on Medical Education).

Association for Medical Education in Europe (1999) A Critical Appraisal of Medical Education. Abstracts of AAME Conference, Linkpoing, Sweden, 29 August to 1 September 1999 (Dundee, Scotland, AMEE).

Association for Medical Education in Europe (1996) AMEE Education Guide No. 7: Task-based Learning: An Educational Strategy for Undergraduate, Postgraduate and Continuing Medical Education (Dundee, Scotland, AMEE).

Association for Medical Education in Europe (1999) AMEE Education Guide No. 14: Outcome-based Education (Dundee, Scotland, AMEE).

Australian Medical Council Inc. (1992) The Assessment and 
Accreditation of Medical Schools by the Australian Medical Council (Australian Medical Council Incorporated).

Bandaranayake, R. (2000) The Concept and Practicability of a Core Curriculum in Basic Medical Education, Medical Teacher 22(6), p. 560.

Boelen, C. (1995) Prospects for Change in Medical Education in the Twenty-first Century, Academic Medicine 70(7), p. S21 (WHO/ECFMG Conference, October 3-6, 1994, Geneva, Switzerland).

Branch, W.T. (2000) The Ethics of Caring and Medical Education, Academic Medicine 75(2), p. 127.

CanMEDS 2000 (1996) Project Skills for the new millennium: report of the societal needs working group, The Royal College of Physicians and Surgeons of Canada's Canadian Medical Education Directions for Specialists 2000 Project (Ottawa, Ontario, Canada).

CanMEDS 2000 (2000) Extract from the CanMEDS 2000 Project Societal Needs Working Group Report (2000), Medical Teacher 22(6), p. 549.

Chaves, M.M. et al. (1984) Cambios en la education medica. Analisis de la integracion docente asistencial en America Latina (Caracas, Venezuela, Federacion Pana-mericana de Asociaciones de Facultades y Escuelas, No. 3).

DeAngelis, C.D. (Ed.) (1999) The Johns Hopkins University School of Medicine Curriculum for the Twenty-first Century (Baltimore, The Johns Hopkins University Press).

DelVecchio Good, M. (1995) American Medicine: The Quest for Competence (Berkeley, University of California Press).

Education Committee of the General Medical Council (1993) Tomorrow's Doctors: Recommendations on Undergraduate Medical Education (London, General Medical Council).

Eitel, F. and Steiner, S. (1999) Evidence-based learning, Medical Teacher 21(5), p. 506.

Federacion Panamericana de Asociaciones de Facultades (Escuelas) de Medicina No. 13 (1986) Cooperacion internacional para el desarrollo de la educacion medica (Caracas, Venezuela).

Federacion Panamericana de Asociaciones de Facultades (Escuelas) de Medicina No. 17 (1990) Medical Education in the Americas. The challenge of the nineties, Final Report of the EMA project. p. 240 (Caracas, Venezuela).

Flexner, A. (1910) Medical Education in the United States and Canada. A Report to the Carnegie Foundation for the Advancement of Teaching. (Boston, MA, D.B. Updike, The Merrymount Press).

Gastel, B.A. (1995) Toward a Global Consensus on Quality Medical Education: Serving the Needs of Populations and Individuals: Summary of the Consultation, Academic Medicine, 70(7), p. S3 (WHO/ECFMG Conference, 3-6 October, 1994, Geneva, Switzerland).

General Medical Council (1998) Good Medical Practice (General Medical Council. London).

Goldie, J. (2000) Review of ethics curricula in undergraduate medical education, Medical Education, 34, p. 108.

Gonnella, J.S., Hojat, M., Erdmann, J.B. (1993) What we have learned, and where do we go from here? Academic Medicine, 68(2), p. S79.

Halpern, R. et al. (2001) A Synthesis of Nine Major Reports on Physicians' Competencies for the Emerging Practice Environment, Academic Medicine, 76(6), p. 606.

Hamilton, J.D., Vandewerdt, J.M. (1990) The accreditation of undergraduate medical education in Australia, Medical Journal of Australia, 153, p. 541.

Hamilton, J.D. (1995) Establishing Standards and Measurement Methods for Medical Education, Academic Medicine, 70(7), p. S51.

Henry, R. (1997) Undergraduate programme objectives: the basis for learning and assessing by domain, in: $\mathrm{R}$. Henry, K. Byrne and C. Engel (Eds.) Imperatives in Medical Education, pp. 18-23 (Callaghan, NSW., Faculty of Medicine and Health Sciences, The University of Newcastle).

Institut d'Estudis de la Salut (1997) Professional Competencies on Health Sciences. Future scenario for the health professionals (Barcelona, Spain).

Karle, H., Nystrup, J. (1996) Evaluation of Medical Specialist Training: Assessment of Individuals and Accreditation of Institutions. Association for Medical Education in Europe Occasional Paper No. 1 (Dundee, Scotland, Centre for Medical Education).

Kern, D.E. et al. (1998) Curriculum Development for Medical Education: A Six-Step Approach (Baltimore, The Johns Hopkins University Press).

Lloyd, J.S. (Ed.) (1982) Evaluation of noncognitive skills and clinical performance (American Board of Medical Specialties, Chicago, Illinois).

Ludmerer, K.M. (1999) Time to Heal - American Medical Education from the Turn of the Century to the Era of Managed Care. (New York, Oxford University Press).

Medical School Objectives Working Group (1999) Learning Objectives for Medical Student EducationGuidelines for Medical Schools: Report I of the Medical School Objectives Project. Academic Medicine, 74(1), p. 13.

National Association of Medical Students (1994) Today's Students on "Tomorrow's Doctors" A Guide to Implementing the General Medical Council Recommendations on Undergraduate Medical Education (National Association of Medical Students). 
O’Neill, P.A., Metcalfe, D., and David, T.J. (1999) The core content of the undergraduate curriculum in Manchester, Medical Education, 33, p. 121.

Paice, E. (Ed.) (1998) Delivering the New Doctor. (Edinburgh, Scotland, Association for the Study of Medical Education).

Rabinowitz, H.K. et al. (2001) Innovative approaches to educating medical students for practice in a changing health care environment: the National UME-21 Project, Academic Medicine, 76(6), p. 587.

Sajid, A. et al. (Eds.) (1994) International Handbook of Medical Education (Westport, CT, Greenwood Press).

Schwarz, R.M. (1998) On moving towards international standards in health professions education. Changing Medical Education and Medical Practice (WHO, Geneva, Switzerland).

Scott, C.S. et al. (1991) Clinical behavior and skills that faculty from 12 institutions judged were essential for medical students to acquire, Academic Medicine, 66(2), p. 106.

Sprafka, S. (1999) Defining and using professional behavior standards: an approach underway at the University of New England, Education for Health, 12(2), p. 245.

Stobo, J.D., Blank, L.L. (1998) Project Professionalism: Staying Ahead of the Wave (American Board of Internal Medicine, Philadelphia, Pennsylvania).

Swanson, A.G., Anderson, M.B. (1993) Educating medical students, assessing change in medical education - the road to implementation, Academic Medicine, 68(6), Supplement.

Tosteson, D.C., Adelstein, S.J., Carver, S.T. (Eds.) (1994) New Pathways to Medical Education (Cambridge, MA, Harvard University Press).

University of Dundee Medical School (n.d.) Teaching Tomorrow's Doctors in Dundee - A five-year strategy
1999-2003 (Dundee, Scotland, University of Dundee Medical School).

U.S. Department of Health and Human Services (1992) Council on Graduate Medical Education, Third Report: Improving Access to Health Care Through Physician.

Wojtczak, A. and Schwarz, M.R. (2000a) International standards and medical education: discussion document.

Wojtczak, A. and Schwarz, M.R. (2000b) Minimum essential requirements and standards in medical education, Medical Teacher, 22(6), p. 555.

World Federation for Medical Education Executive Council (1998) International standards in medical education: assessment and accreditation of medical schools, Medical Education, 32, p. 549.

World Federation for Medical Education Task Force (2000) Defining international standards in basic medical education, Report of the Working Party, Copenhagen 1999, Medical Education, 34(8), p. 665.

World Federation for Medical Education (1994) Proceedings of the World Summit on Medical Education. Ed. Henry Walton, Medical Education, Vol. 28, Suppl. 1.

World Health Organization (1992) Towards the Assessment of Quality in Medical Education (Geneva, Switzerland, WHO).

World Health Organization (1993) Increasing the Relevance of Education for Health Professionals Report of a WHO Study Group on Problem-solving Education for the Health Professionals (Geneva, Switzerland, WHO).

World Health Organization (1996) Doctors for health: A WHO Global Strategy for Changing Medical Education and Medical Practice for Health for All. (Geneva, Switzerland). 